\title{
Review: combining pindolol with an SSRI improves early outcomes in people with depression
}

Ballesteros J, Callado LF. Effectiveness of pindolol plus serotonin uptake inhibitors in depression: a meta-analysis of early and late outcomes from randomised controlled trials. J Affect Disord 2004;79:137-47.

\section{What is the efficacy and tolerability of pindolol plus selective serotonin reuptake inhibitors (SSRIs) in people with depression?}

\section{METHODS}

-

Design: Systematic review with meta-analysis

Data sources: MEDLINE (1966-2001), EMBASE (1980-2001)

PsyclNFO (1967-2001), Current Contents (1995-2001), plus

hand searches of reference lists and reviews.

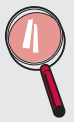

Study selection and analysis: Eligible studies were randomised controlled trials (RCTs) comparing pindolol plus SSRIs with placebo plus SSRIs in people with depression. RCTs examining early response (between 10 days and 2 weeks) and late response (3-6 weeks) were included. Studies involving non-SSRI antidepressants and crossover RCTs were excluded. Sensitivity and heterogeneity analyses were conducted.

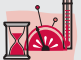

Outcomes: Depressive symptoms: efficacy assessed by the number of participants responding to treatment (defined as a decrease of $\geqslant 50 \%$, or similar criterion, on the Hamilton Depression Rating Scale (HDRS) or Montgomery-Åsberg Depression Rating Scale (MADRAS)). Tolerability: proportion of the total study population not completing the study. Safety: proportion of total participants experiencing side effects.

\section{MAIN RESULTS}

Nine RCTs met inclusion criteria (594 participants).

Early response: five RCTs met inclusion criteria. Pindolol plus SSRIs significantly improved depressive symptoms compared with placebo plus SSRIs, for the first two weeks of treatment (OR 2.8, 95\% CI 1.4 to 5.7 ; $\mathrm{NNT}=6,95 \%$ CI 4 to 20 ).

Late response: seven RCTs met inclusion criteria. There were no significant differences between groups after 3-6 weeks (OR 1.4, $95 \%$ CI 0.8 to 2.7). However, the late response studies were heterogeneous.

Tolerability and safety: there were no significant differences in tolerability or adverse events between groups (pindolol plus SSRIs $v$ placebo plus SSRIs; OR for tolerability, $1.3,95 \%$ CI 0.8 to 2.3; OR for adverse events, $1.3,95 \%$ CI 0.7 to 2.1 ).

\section{CONCLUSIONS}

During the first two weeks of treatment, the addition of pindolol to an SSRI appears to increase response; however there was no evidence of improved efficacy beyond this period.

For correspondence: Javier Ballesteros, Department of Neuroscience, Medical Psychology and Psychiatry, University of the Basque Country, Barrio Sarriena, Leioa, Spain; onpbaroj@lg.ehu.es

Sources of funding: none specified.

\section{Commentary}

A mid all the current arguments about the mechanism and efficacy of antidepressants, virtually everyone can agree that there is a delay-the antidepressant "latency"-before the specific antidepressant effect is clear. It is common for the antidepressant effect to be delayed. The formulation of a rapidly acting antidepressant would be a major landmark. Manufacturers' claims of rapid onset for their particular drug should be regarded with caution, as clinical trials are not usually designed with time of onset in mind.

The problem is complicated because we do not know why latency occurs. Selective serotonin reuptake inhibitors (SSRI) selectively inhibit serotonin reuptake by the pre-synaptic neuron immediately; but there is commonly a lag of 3-4 weeks before they exert an antidepressant effect. There have been several suggested mechanisms, but as yet no unifying theory.

In the mid-1990s, Artigas et $a l^{1}$ and Blier et $a l^{2}$ suggested that continued activity of presynaptic $5 \mathrm{HT} 1 \mathrm{~A}$ autoreceptors was responsible for antidepressant latency, and that specific blockade of these receptors by the $\beta$-antagonist pindolol could reduce this latency-and so it proved in open label studies. The key was of course to test the effect in randomised controlled studies.

Results have been mixed. Roughly speaking, in European hands (including our $\mathrm{own}^{3}$ ), the addition of pindolol from the outset of antidepressant (not only with $\mathrm{SSRI}^{4}$ ) treatment does indeed seem to accelerate the antidepressant response in many patients, especially if they have not been treated for depression before (the severity of the depression seems to be less important). However, some US studies ${ }^{5}$ have been less successful, as have studies looking at treatment resistance. So the true place of pindolol augmentation remains debated, with the honours so far in favour of the efficacy of the combination. This metaanalysis is a helpful synthesis of knowledge up to 2002

The clinical advice would be that pindolol augmentation is worth trying in new patients, as long as $\beta$ blockers are not otherwise contraindicated, and may lead to dramatic and rapid clinical improvements. There seems to be no point in giving pindolol for more than 2-3 weeks.

Michael T Isaac MA, MRCPsych Consultant Psychiatrist, South London \& Maudsley NHS Trust and Senior Lecturer, GKT Medical School \& Institute of Psychiatry, University of London, UK

1 Artigas F, Perez V, Alvarez E. Pindolol induces a rapid improvement of depressed patients treated with serotonin reuptake inhibitors. Arch Gen Psychiatry 1994;51:248-51.

2 Blier P, Bergeron R. Effectiveness of pindolol with selected antidepressant drugs in the treatment of major depression. J Clin Psychopharmacol 1995; 15:217-22.

3 Tome MB, Isaac MT, Harte R, et al. Paroxetine and pindolol: a randomized trial of serotonergic autoreceptor blockade in the reduction of antidepressant latency. Int Clin Psychopharmacol 1997; 12:81-9.

4 Isaac MT, Isaac MB, Gallo F, et al. Milnacipran and pindolol: a randomised trial of reduction of antidepressant latency. Hum Pychopharmacol 2003; 18:595-601.

5 Berman RM, Darnell AM, Miller HL, et al. Effect of pindolol in hastening response to Fluoxetine in the treatment of major depression: a double-blind, placebo-controlled trial. Am J Psychiatry 1997;154:37-43. 